\title{
Estimativa da permeabilidade de arenitos a partir de propriedades petrofísicas e elásticas
}

Simonária Siqueira Fidelis, Marco Antônio Rodrigues de Ceia, Roseane Marchezi Missagia, Irineu Lima Neto. Laboratório de Engenharia e Exploração de Petróleo (LENEP) - Universidade Estadual do Norte Fluminense (UENF)

Copyright 2016, SBGf - Sociedade Brasileira de Geofísica

Este texto foi preparado para a apresentação no VII Simpósio Brasileiro de Geofísica Ouro Preto, 25 a 27 de outubro de 2016. Seu conteúdo foi revisado pelo Comitê Técnico do VII SimBGf, mas não necessariamente representa a opinião da SBGf ou de seus associados. E proibida a reprodução total ou parcial deste material para propósitos comerciais sem prévia autorização da SBGf.

\section{Abstract}

The aim of this study is to estimate the permeability through petrophysical (clay content and porosity) and elastic (velocities, attenuation coefficient of $P$ and $S$ waves) properties. Empirical relationships have been proposed using multivariate analysis to estimate the permeability in dry sandstone rocks on atmospheric pressure conditions. The results show good correlations, and the best result displays an adjusted correlation coefficient $R^{2}=0.86$. To realization of this study were used data from MARTIN (1996).

\section{Introdução}

O hidrocarboneto é uma fonte de energia não renovável de grande importância e o total de energia consumido anualmente é aproximadamente $40 \%$ da matriz energética (FIGUEIREDO, 2015). Essa demanda justifica a grande importância do investimento e melhorias em métodos para caracterização de reservatórios, almejando otimizar tempo e custo de produção. O óleo é encontrado nas rochas-reservatório e são caracterizadas por bons valores de porosidade e permeabilidade (ROSA, 2006). As rochas-reservatório são importantes para determinar e entender as propriedades petrofísicas e elásticas em laboratório. A permeabilidade é definida como a habilidade que uma formação rochosa tem de conduzir fluidos, assim é uma propriedade petrofísica de grande interesse, porém, por não ser medida diretamente, é um dos parâmetros mais difíceis de ser estimado em um estudo de reservatório.

Tentativas para estimar a permeabilidade através de propriedades petrofísicas e elásticas foram feitas em varias pesquisas (KLIMENTOS 1991; MARTIN 1996; PRASAD 2003). KLIMENTOS (1991) fez um estudo sobre a correlação entre a onda compressional (onda P) e a permeabilidade, incluindo outros fatores como porosidade e conteúdo de argila, encontrando valores de $\mathrm{R}^{2}$ de 0.93 e 0.96 . Este mesmo autor, concluiu que 0 efeito da permeabilidade na velocidade da onda $P$ é negligenciável, sugerindo a inclusão de mais parâmetros na correlação. MARTIN (1996) fez correlações entre permeabilidade, e atenuação da onda $P$ e $S$, suas melhores correlações para estimar a permeabilidade tiveram um $\mathrm{R}^{2}$ entre 0.54 e 0.56 . PRASAD (2003) correlacionou permeabilidade e velocidade da onda $P$, agrupando dados de porosidade e permeabilidade de acordo com seus valores de índice de zona de fluxo, resultando um bom $\mathrm{R}^{2}$ de 0.92 .

O objetivo deste trabalho é determinar uma relação empírica que melhor estime a permeabilidade através das propriedades petrofísicas e elásticas. Para determinar essa relação será utilizada técnica de regressão múltipla linear.

Os dados laboratoriais usados neste estudo foram retirados de MARTIN (1996), são amostras de arenitos da formação de Milk River da província de Writing-onstone park. Um total de 60 amostras de arenitos foram estudadas. Velocidade e atenuação foram obtidas usando o método de transmissão ultrassônica, utilizando uma frequência de $1.0 \mathrm{MHz}$ para ondas $\mathrm{P}$ e $\mathrm{S}$. A velocidade e atenuação foram medidas com as amostras secas sobre condições de pressões atmosféricas. O conteúdo de argila foi determinado pelo método de difração de raios- $X$, a permeabilidade usando um permeâmetro a gás hélio e a porosidade a partir do porosímetro a gás.

\section{Metodologia}

A análise de regressão é uma metodologia estatística que utiliza a relação entre duas ou mais variáveis quantitativas (ou qualitativas) de tal forma que uma variável pode ser predita a partir da outra ou outras. Esta relação é apresentado por um modelo matemático que relaciona a variável dependente com as variáveis independentes.

A análise de regressão múltipla é um método estatístico que prevê valores de uma ou mais variáveis dependentes através de uma variável independente (ARCHILHA, 2015). A Eq. 1 representa o formato geral da equação de regressão múltipla linear:

$Y=B+B_{1} X_{1}+B_{2} X_{2}+\cdots$

Em que, $Y$ é a variável dependente, $B_{n}$ são constantes determinadas pela regressão linear e $X_{n}$ são as variáveis independentes.

O objetivo é determinar como o modelo estatístico apresentado na Eq. 1 se ajusta aos dados experimentais, a partir da análise do coeficiente de determinação ajustado $\left(R^{2 \prime}\right)$ (Eq. 2), onde $n$ é o número de amostras e $\mathrm{p}$ o número de variáveis do modelo estatístico. O coeficiente de determinação $\mathrm{R}^{2}$ tende a aumentar com a adição de novas variáveis fornecidas ao modelo estatístico (FIGUEIREDO, 2015).

$R^{2 \prime}=1-\left(1-R^{2}\right) \frac{n-1}{n-p-1}$ 
O número de amostras estudadas em uma regressão linear múltipla deve ser grande o suficiente para assegurar coeficientes estáveis. Se o número de amostras for pequeno, a equação gerada não poderá ser generalizada, sendo assim, serão válidas apenas para o conjunto de dados estudado. As equações podem ser generalizadas se a razão do tamanho de dados e números de variáveis for de 10:1 (MILLER \& KUNCE, 1973)

\section{Resultados}

A figura 1 mostra a correlação entre o logaritmo natural da permeabilidade e porosidade para todas as amostras, onde a linha de tendência que melhor se ajustou aos dados foi a de potência, obtendo um coeficiente de determinação $\left(R^{2}\right)$ de 0.5317 . Á Figura 2 correlaciona o logaritmo natural da permeabilidade versus conteúdo de argila para 8 amostras, e o melhor ajuste foi 0 de potência com $\mathrm{R}^{2}$ de 0.2175 , não apresentando uma boa correlação entre permeabilidade e conteúdo de argila. Figuras 3 e 4 correlacionam logaritmo natural da permeabilidade versus velocidade da onda $P$ e velocidade da onda $S$, respectivamente. A linha de tendência que melhor se ajustou em ambos foi a polinomial, com $\mathrm{R}^{2}$ de 0.5034 e 0.5034 . Figura 5 e 6 mostram a correlação entre o logaritmo natural da permeabilidade versus atenuação das ondas $P$ e $S$ com $\mathrm{R}^{2}$ de 0.0378 e 0.0928 , respectivamente. Ambos tiveram o melhor ajuste com a linha de tendência polinomial. Figuras 7 e 8 mostram as correlações entre o logaritmo natural da permeabilidade versus coeficiente de atenuação das ondas $P$ e $S$ e exibem $R^{2}$ de 0.5211 e 0.4682 , respectivamente. A melhor tendência para a figura 7 foi logarítmica, e para a figura 8 polinomial.

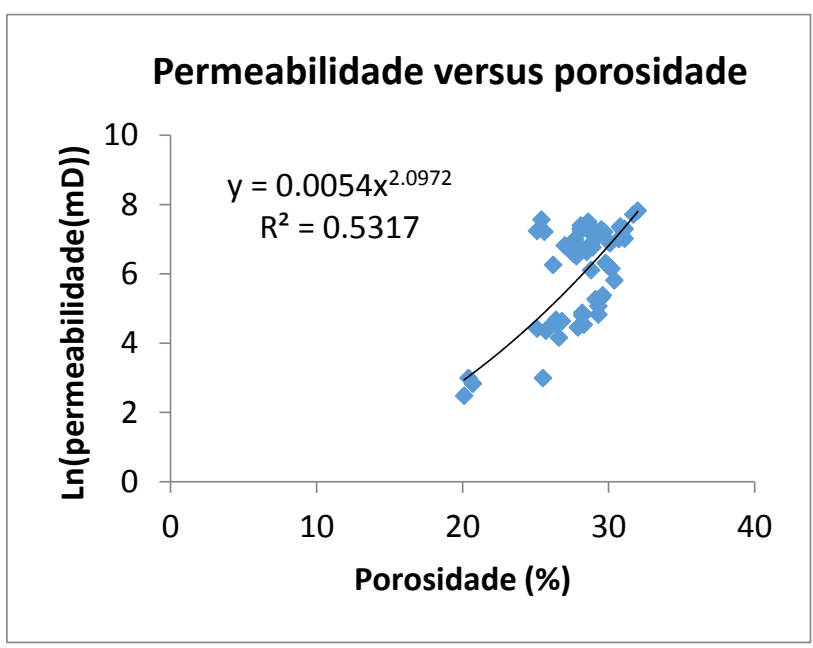

Figura 1 - Logaritmo natural da permeabilidade versus porosidade.

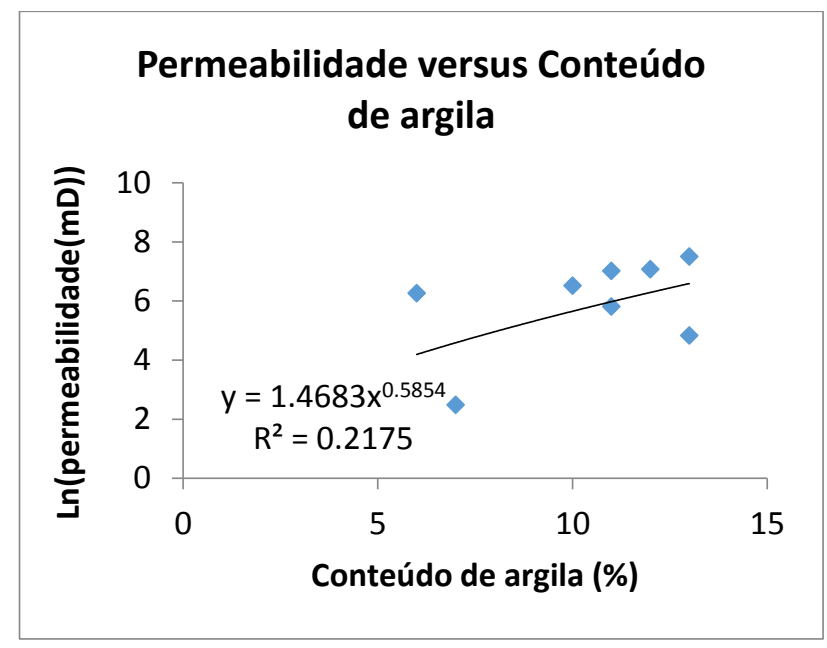

Figura 2 - Logaritmo natural da permeabilidade versus conteúdo de argila.

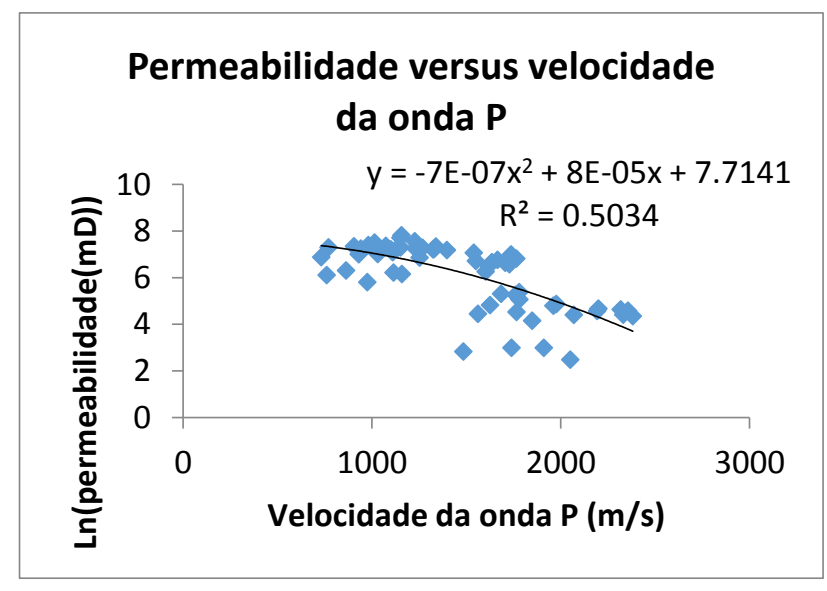

Figura 3 - Logaritmo natural da permeabilidade versus velocidade da onda $P$.

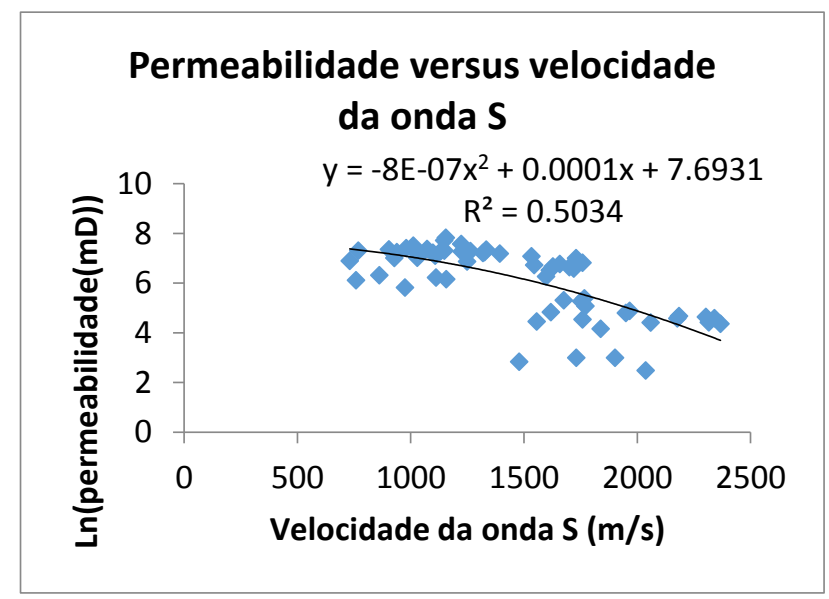

Figura 4 - Logaritmo natural da permeabilidade versus velocidade da onda $\mathrm{S}$. 


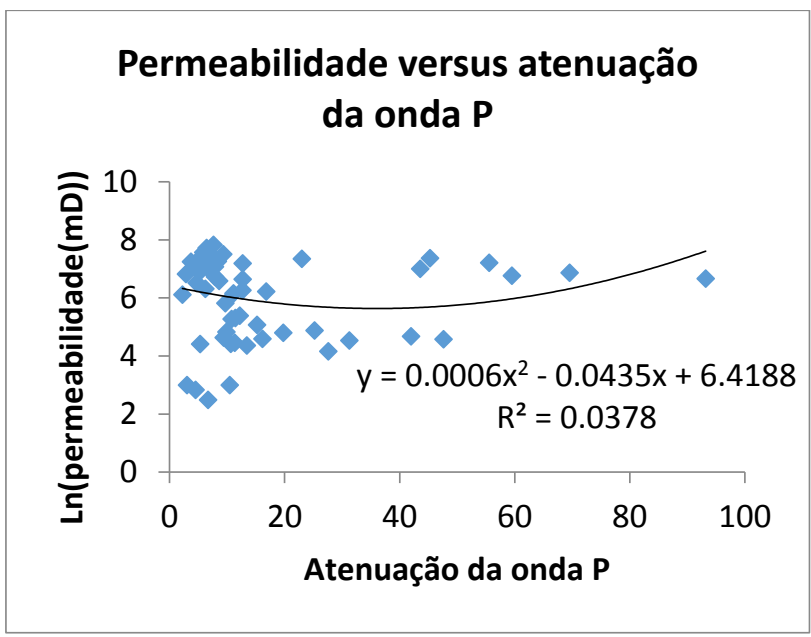

Figura 5 - Logaritmo natural da permeabilidade versus atenuação da onda $P$.

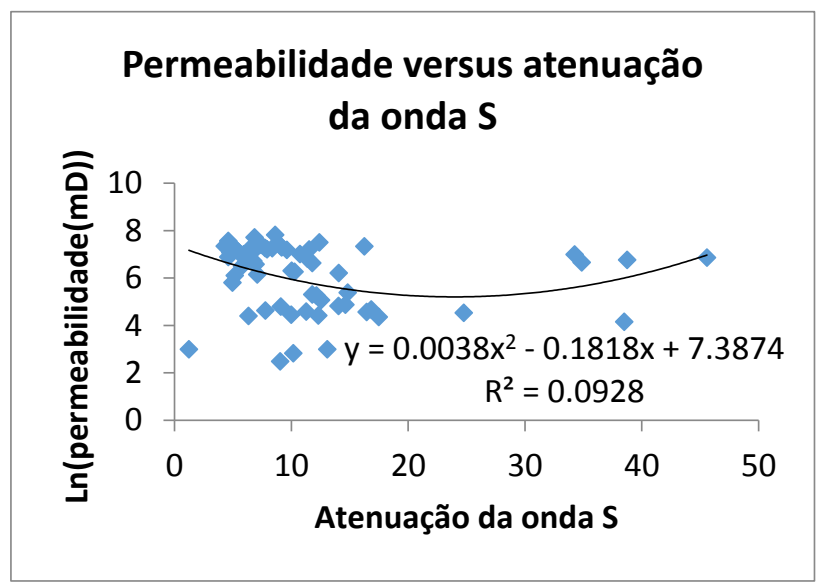

Figura 6 - Logaritmo natural da permeabilidade versus atenuação da onda $S$

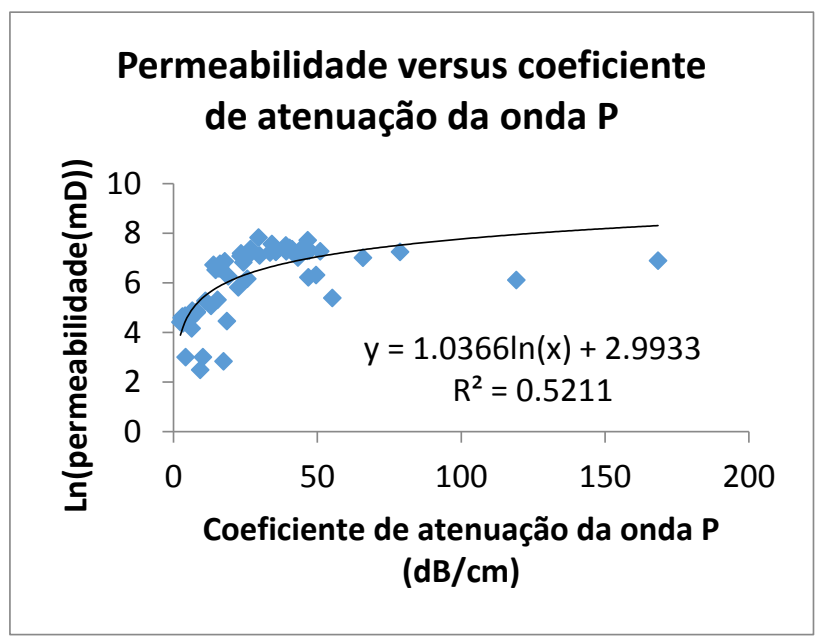

Figura 7 Logaritmo natural da permeabilidade versus coeficiente de atenuação da onda $P$.

\section{Permeabilidade versus coeficiente de atenuação da onda $S$}

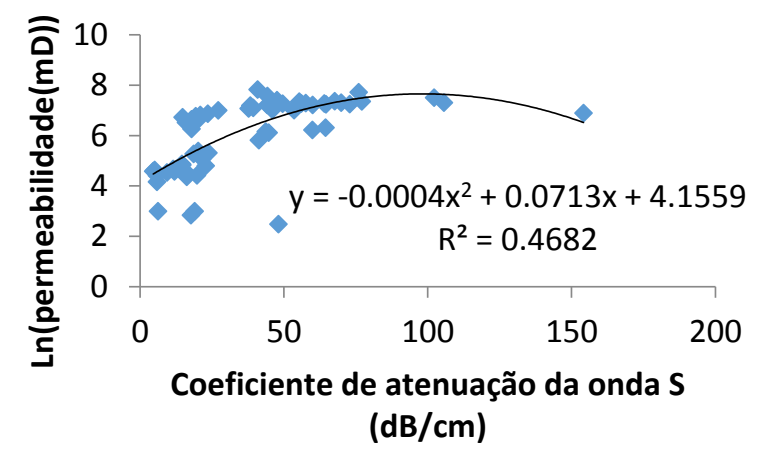

Figura 8 - Logaritmo natural da permeabilidade versus coeficiente de atenuação da onda S.

Várias regressões múltiplas e lineares foram realizadas para os dados do presente trabalho. As regressões foram separadas em grupo I e grupo II. O grupo I não leva em conta o conteúdo de argila e o grupo II leva em conta o mesmo. Apenas 8 amostras possuíam dados de conteúdo de argila, logo as regressões múltiplas do grupo II são baseadas em informações destas. As regressões múltiplas do grupo I são baseadas em informações de todas as 60 amostras de arenitos. As equações que expressaram os melhores resultados para o grupo I estão representadas nas Eqs. 3 a 5, e as do grupo II nas Eqs. 6 a 8.

Também foram feitos gráficos da permeabilidade estimada versus permeabilidade medida, todos em escala logarítmica (figuras 9 a 14), mostrando então a eficácia de cada um dos modelos apresentados nas Eqs. 3 a 8 . Assim, é possível perceber de maneira mais clara os resultados dos modelos propostos neste trabalho.

A fim de avaliar a confiabilidade dos ajustes propostos nas Eqs. 3 a 5 para um número menor de amostras, foi feito um teste com apenas oito amostras, das quais são as mesmas utilizadas nos modelos das Eqs. 6 a 8 (grupo II), porém, sem considerar o conteúdo de argila. Os novos coeficientes de determinação ajustados foram respectivamente $0.56,0.56$ e 0.62 . Observa-se que os novos coeficientes de determinação ajustados com informações de 8 amostras, estão bem próximos dos antigos, a qual levava em consideração informações de 60 amostras, isto mostra que o modelo é confiável. Os resultados são representados nas figuras 15 a 17.

$K=-3,1585+0,3025 \emptyset-0,0034 \alpha P+0,0216 \alpha S R^{2^{\prime}}=$

0,55

$K=0,8298+0,0066 \alpha S+0,2410 \emptyset-0,0012 V s \quad R^{2^{\prime}}=$ 0,61 
$K=-0,8387-0,0054 \alpha P+0,0143 \alpha S+0,2478 \varnothing-$

$0,1423 V p+0,1426 V s \quad R^{2^{\prime}}=0,62$

$K=-2,774+0,305 \emptyset+0,053 \alpha P-0,093 C \quad R^{2^{\prime}}=$ 0,53

$K=-39,84+1,42 \varnothing+0,0075 V s+0,085 \alpha S-$

$0,7675 C R^{2^{\prime}}=0,77$

$K=-34,58-0,601 C+1.11 \emptyset-0,487 V p+0,084 \alpha s+$

$0,49 V S \quad R^{2^{\prime}}=0,86$

Onde, $K, \quad \varnothing, \quad C, \quad V p, \quad V s, \quad \alpha P, \quad \alpha S$ e $R^{2 \prime}$, são respectivamente: Permeabilidade, porosidade, conteúdo de argila, velocidade da onda $P$, velocidade da onda $S$, coeficiente de atenuação da onda $P$, coeficiente de atenuação da onda $S$ e coeficiente de determinação ajustado.

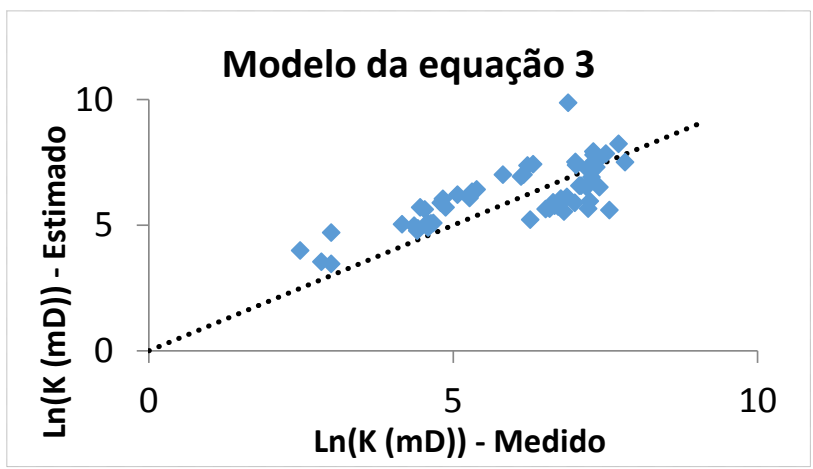

Figura 9 - Logaritmo natural da permeabilidade estimada versus permeabilidade medida do modelo da Eq. 3, referente a 60 amostras.

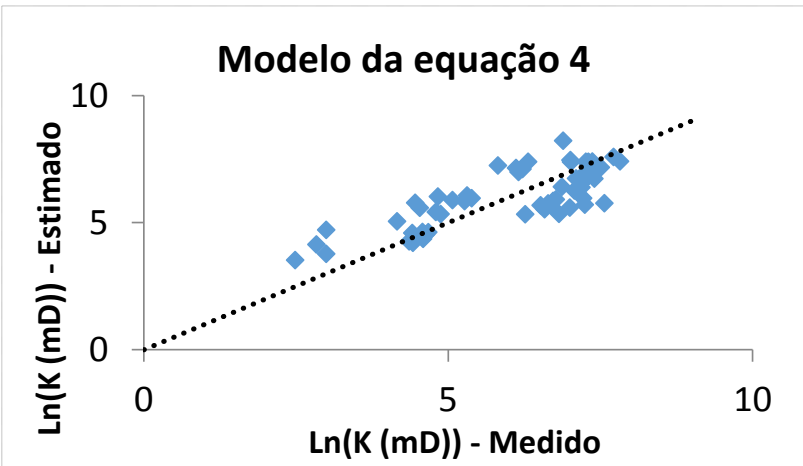

Figura 10 - Logaritmo natural da permeabilidade estimada versus permeabilidade medida do modelo da Eq. 4, referente a 60 amostras.

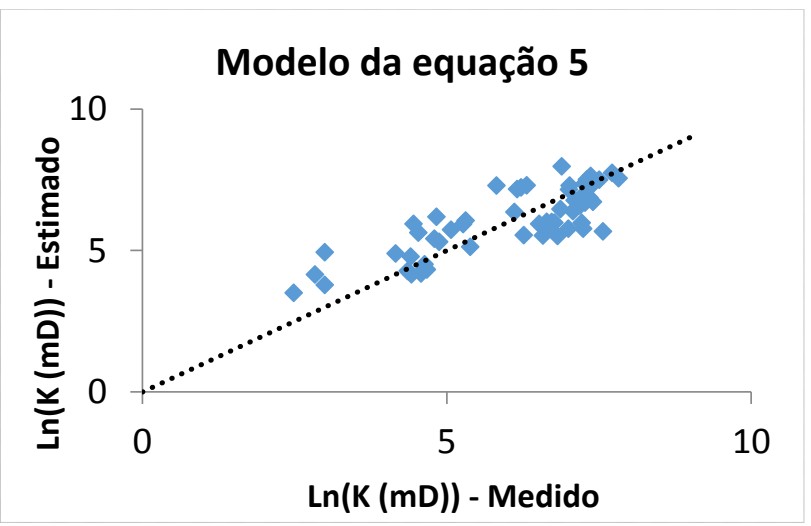

Figura 11 - Logaritmo natural da permeabilidade estimada versus permeabilidade medida do modelo da Eq. 5, referente a 60 amostras.

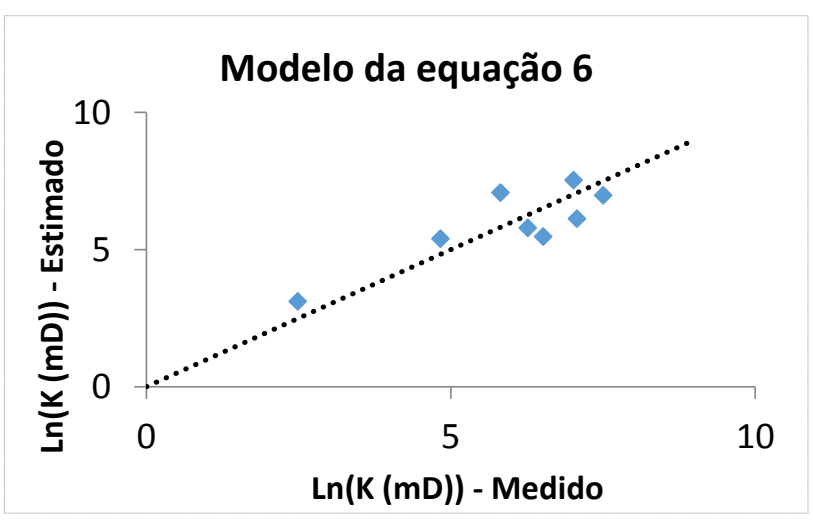

Figura 12 - Logaritmo natural da permeabilidade estimada versus permeabilidade medida do modelo da Eq. 6, referente a 8 amostras.

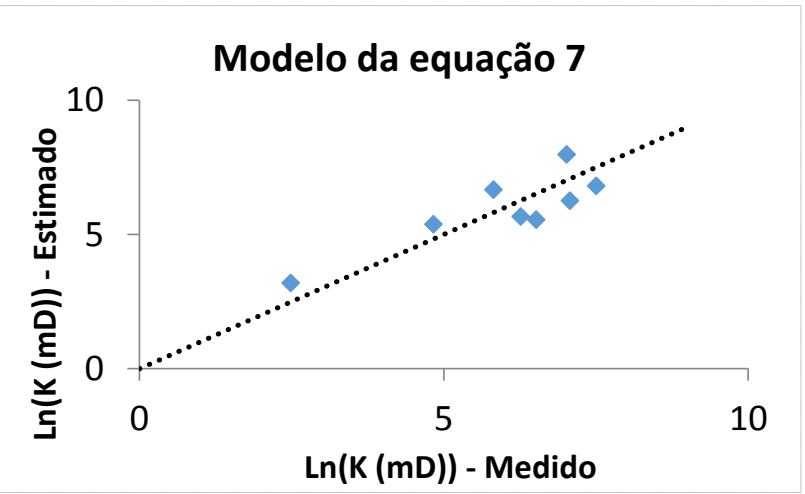

Figura 13 - Logaritmo natural da permeabilidade estimada versus permeabilidade medida do modelo da Eq. 7, referente a 8 amostras. 


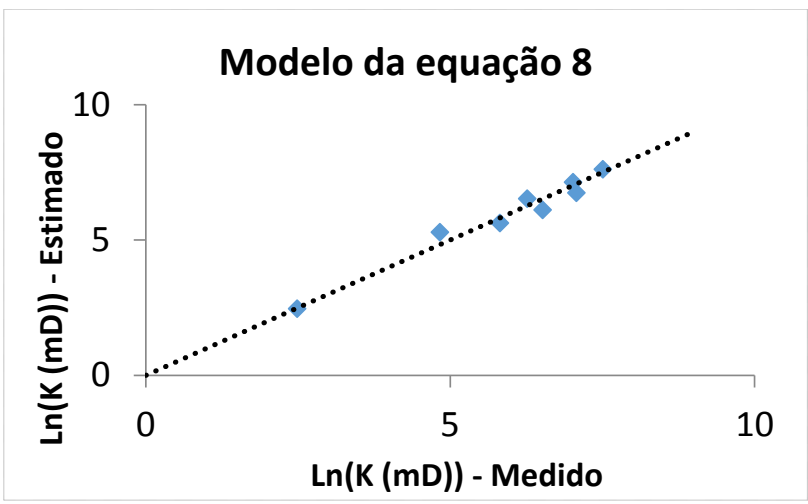

Figura 14 - Permeabilidade estimada versus permeabilidade medida do modelo da Eq. 8, referente a 8 amostras.

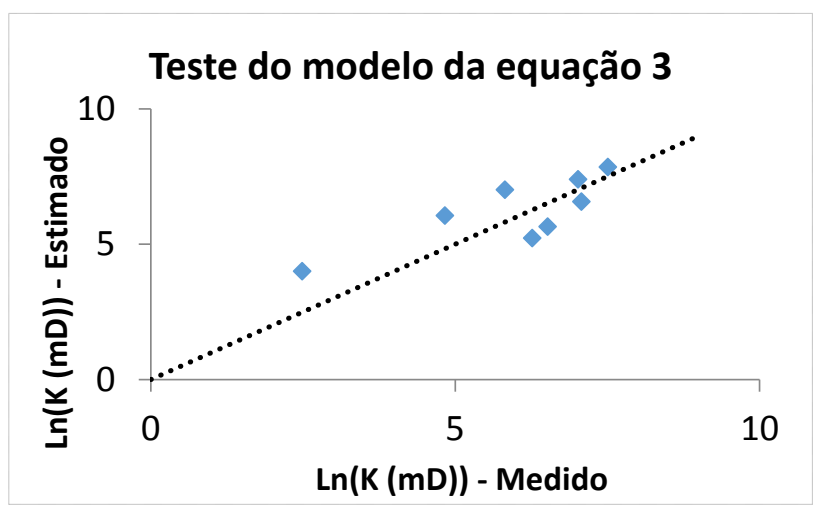

Figura 15 - Teste do modelo da Eq. 3 para 8 amostras, resultando em um $R^{2^{\prime}}$ de 0,56 .

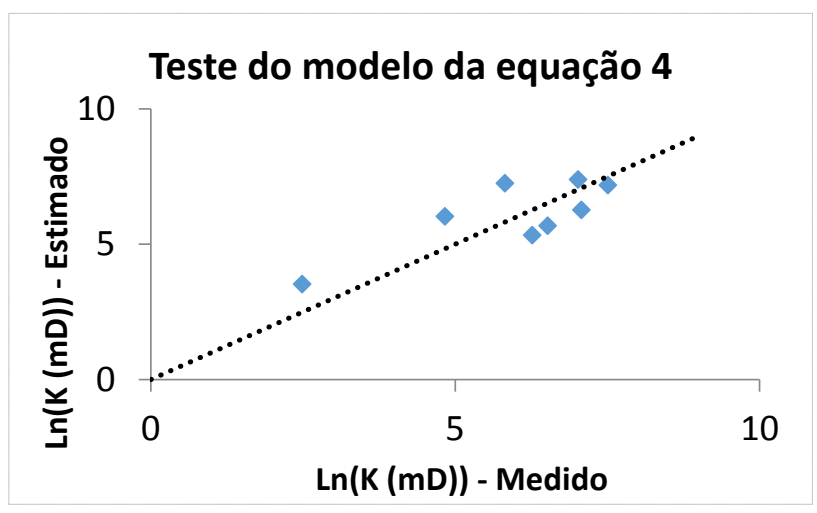

Figura 16 - Teste do modelo da Eq. 4 para 8 amostras, resultando em um $R^{2^{\prime}}$ de 0,56 .

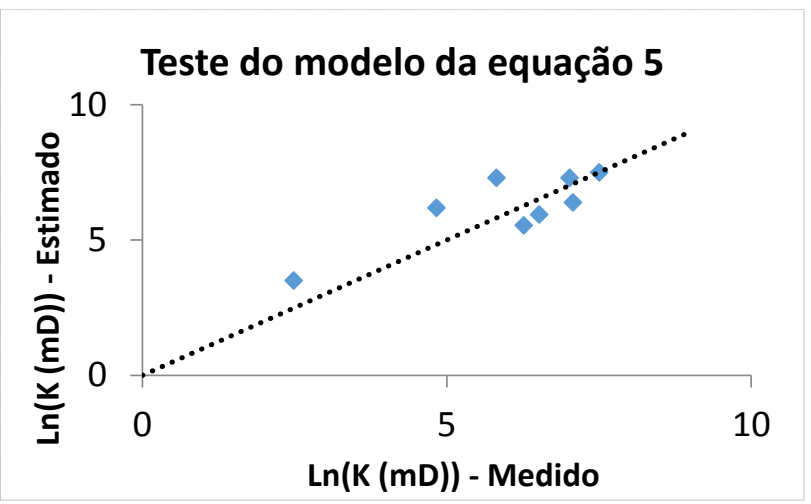

Figura 17 - Teste do modelo da Eq. 5 para 8 amostras, resultando em um $R^{2^{\prime}}$ de 0,62 .

Os modelos que melhor estimaram a permeabilidade são os apresentados nas Eqs. 5 e 8, com $R^{2^{\prime}}$ de 0,62 e 0,86, respectivamente. No entanto, a fim de avaliar a contribuição de cada propriedade do espaço poroso presente nas Eqs. 5 e 8, foi realizada uma análise de sensibilidade que consiste em retirar uma determinada propriedade da equação analisada e verificar a sua influência através do coeficiente de determinação ajustado. A tabela 1 mostra as novas equações geradas para analisar a contribuição das propriedades do espaço poroso da Eq. 5, levando em consideração as 60 amostras. Como observado, a Eq. 9 presente na tabela 1 possui as mesmas propriedades do que a Eq. 5, exceto a porosidade, resultando então em um coeficiente de determinação ajustado de 0,47. A Eq. 10 mostra a retirada da propriedade $\alpha S$, obtendo um $R^{2^{\prime}}$ de 0,60 , e a Eq. 11 teve $\alpha P$ retirado, resultando em um $R^{2^{\prime}}$ de 0,62 , a Eq. 12 teve $V p$ retirado e o $R^{2^{\prime}}$ foi de 0,61 e na Eq. 13 o $V s$ foi retirado, resultando em um $R^{2^{\prime}}$ de 0,61 .

A tabela 2 mostra o mesmo tipo de análise apresentada na tabela 1, porém dessa vez referente à Eq. 8, que leva em consideração 8 amostras. Na Eq. 14 da tabela 2 estão presentes as mesmas propriedades da Eq. 5, exceto $\alpha S$, resultando em um $R^{2^{\prime}}$ de 0,29. A Eq. 15 mostra a retirada da propriedade $V s$, obtendo um $R^{2^{\prime}}$ de 0,77 , na equação $16 \circ \mathrm{Vp}$ foi retirado e o $R^{2^{\prime}}$ foi de 0,77 , na equação 17 a propriedade $\varnothing$ foi retirada, obtendo $R^{2^{\prime}}$ de 0,49 e por último, na Eq. 18 foi retirada a propriedade $C$, e o $R^{2^{\prime}}$ foi de 0,61 .

Tabela 1- Análise de sensibilidade da Eq. 5.

\begin{tabular}{|c|c|}
\hline \multicolumn{1}{|c|}{ Equação } & $\begin{array}{c}\text { Coeficiente } \\
\text { ajustado de } \\
\text { determinação } \\
\left(R^{2^{\prime}}\right)\end{array}$ \\
\hline $\begin{array}{l}K=7,53-0,11 V p+0,11 V S-0,004 \alpha P \\
+0,011 \alpha S\end{array}$ & 0,47 \\
\hline$K=1,02-0,08 V p+0,08 V S-0,0006 \alpha P$ & 0,60 \\
\hline
\end{tabular}




\begin{tabular}{|l|r|}
\hline$+0,239 \emptyset$ & $(10)$ \\
$\begin{array}{l}K=-1,32-0,17 V p+0,17 V s-0,012 \alpha S \\
+0,246 \emptyset\end{array}$ & 0,62 \\
\hline $\begin{array}{l}K=1,03-0,001 V s-0,011 \alpha S- \\
0,008 \alpha P+0,244 \varnothing\end{array}$ & 0,61 \\
\hline $\begin{array}{l}K=1,02-0,001 V p-0,011 \alpha S- \\
0,008 \alpha P+0,244 \varnothing\end{array}$ & (12) \\
\hline
\end{tabular}

Tabela 2- Análise de sensibilidade da Eq. 8.

\begin{tabular}{|l|c|}
\hline \multicolumn{1}{|c|}{ Equação } & $\begin{array}{r}\text { Coeficiente } \\
\text { ajustado de } \\
\text { determinação } \\
\left(R^{2^{\prime}}\right)\end{array}$ \\
\hline $\begin{array}{l}K=4,46+0,061 \mathrm{C}+0,015 \emptyset-0.51 \mathrm{Vp} \\
+0,51 \mathrm{Vs}\end{array}$ & 0,29 \\
\hline $\begin{array}{l}\mathrm{K}=-39,74-0,767 \mathrm{C}+1,42 \varnothing+0,007 \mathrm{Vp} \\
+0,085 \alpha \mathrm{S}\end{array}$ & $(15)$ \\
\hline $\begin{array}{l}\mathrm{K}=-39,84-0,767 \mathrm{C}+1,42 \varnothing \quad \text { (16) } \\
+0,085 \alpha \mathrm{S}+0,007 \mathrm{Vs}\end{array}$ & 0,77 \\
\hline $\begin{array}{l}\mathrm{K}=-0,471+0,042 \mathrm{C}+0,028 \alpha \mathrm{S} \\
+0,91 \mathrm{Vs}-0,90 \mathrm{Vp}\end{array}$ & (17) \\
\hline $\begin{array}{l}\mathrm{K}=-10,44+0,040 \alpha \mathrm{S}+0,78 \mathrm{Vs}- \\
0,77 \mathrm{Vp}+0,27 \emptyset\end{array}$ & (18)
\end{tabular}

\section{Discussão e Conclusão}

Neste trabalho foi avaliado a correlação existente entre permeabilidade e propriedades petrofísicas e elásticas, cujo objetivo principal era conseguir estabelecer uma função que melhor estimasse a permeabilidade. Posterior às análises, conclui-se que a Eq. 5 foi a que melhor estimou a permeabilidade para o grupo $\mathrm{I}$, com um coeficiente ajustado de 0.62 . Para o grupo II a Eq. 8 foi a que melhor estimou a permeabilidade, com um coeficiente ajustado de 0.86 .

Através de análises conclui-se que um elemento individual não é capaz de estimar a permeabilidade, mas sim, o conjunto de informações deve ser considerado, e que quanto mais informações a respeito do sistema poroso existir, melhor será a estimativa da permeabilidade. Neste sentido, cada propriedade do sistema poroso possui sua contribuição, e por meio da análise de sensibilidade foi possível verificar a influência de cada elemento das Eqs. 5 e 8. A propriedade que teve maior influência na Eq. 5 para estimar a permeabilidade foi a porosidade e em segundo lugar ficou o coeficiente de atenuação da onda S. Já na Eq. 8, a propriedade com maior influência foi o coeficiente de atenuação da onda $S$ e em segundo lugar a porosidade. Logo, conclui-se que as propriedades que mais influenciaram de forma positiva na estimativa da permeabilidade em ambas as equações foram porosidade e coeficiente de atenuação da onda S.
Em contra partida, a propriedade com menor influência na Eq. 5 foi o coeficiente de atenuação da onda $P$, e na Eq. 8 a menor influência verificada foi das velocidades da onda P e S.

Por fim, a metodologia utilizada neste trabalho foi diferente da utilizada por MARTIN (1996) e mostrou equações frente aos resultados com melhores coeficientes de ajustes.

\section{Agradecimentos}

Agradecemos à UENF/LENEP e CAPES pela infraestrutura e aporte financeiro.

\section{Referências}

ARCHILHA, N.L., 2015. Quantificação de parâmetros geométricos do sistema poroso por tomografia de raios $X$ e análise da influência em propriedades. Macaé-RJ, Brasil. Doutorado, Departamento de petrofísica da Universidade Estadual Fluminense, Laboratório de Engenharia de Exploração e Produção de Petróleo, Macaé.

FIGUEIREDO, L.A.B., 2015. Avaliação do sistema poroso e estimativa de permeabilidade utilizando equações modificadas de kozeny em rochas de afloramento. Macaé-RJ, Brasil. Mestrado, Departamento de petrofísica da Universidade Estadual Fluminense, Laboratório de Engenharia de Exploração e Produção de Petróleo, Macaé.

KLIMENTOS, T., 1991. The effects of porositypermeability-clay content on the velocity of compressional waves. Geophysics, 56: 1930-1939.

MARTIN, N.W., 1996. P-Wave and S-wave velocities and attenuations related to permeability Ultrasonic seismic data for sandstone samples from the Writing-on-Stone Provincial Park in Aiberta. Calgary, Canada. Thesis, department of science, University of Calgary, Calgary.

MILLER, D., \& KUNCE, J., 1973. Prediction and statistical overkill revisited. Measurement and Evaluation in Guidance, 6: 157-163.

PRASAD, M., 2003 Velocity-permeability relations within hydraulic units. Geophysics, 68: 108-117.

ROSA, A. J., CARVALHO, R. S., XAVIER, J. A. D., 2006. Engenharia de reservatório de petróleo. Rio de Janeiro, Brasil. 